Communications in Physics, Vol. 28, No. 2 (2018), pp. 155-162

DOI:10.15625/0868-3166/28/2/11367

\title{
STUDYING THE EFFECTS OF VACANCIES ON THE MELTING TEMPERATURE OF AGCE ALLOY
}

\author{
DANG THANH HAI ${ }^{a, \dagger}$, VU VAN HUNG ${ }^{b}$ AND GIANG THI HONG ${ }^{c}$ \\ ${ }^{a}$ Vietnam Education Publishing House \\ ${ }^{b}$ University of Education, Vietnam National Uiversity Hanoi \\ ${ }^{c}$ Faculty of Physics, Hanoi National University of Education \\ ${ }^{\dagger} E$-mail: dthai@nxbgd.vn \\ Received 21 February 2018
}

Accepted for publication 7 May 2018

Published 16 June 2018

\begin{abstract}
In order to evaluate the effects of vacancies on the melting temperature of AgCe alloy, the statistical moment method was used to find out the analytical expressions to determine the Gibbs free energy in the $A B$ substitution alloy and the expression to calculate the melting temperature of perfect and defect $A B$ substitution alloys. The melting temperature was calculated by the numerical calculation method on the perfect and defect AgCe alloys. The calculating results showed that the melting temperature of alloys increases with increasing pressure. The melting temperatures of defect alloys of $\mathrm{AgCe}, \mathrm{Ag}_{2} \mathrm{Ce}$ and $\mathrm{Ag}_{3} \mathrm{Ce}$ are always slightly lower than that of perfect alloys. Especially, the melting temperatures value of the perfect and defect alloys were almost the same at high pressure. The melting temperature of alloys increase with an increase in amount of Ag containing in the alloys. The calculated results agreed well with the experimental results at zero pressure.
\end{abstract}

Keywords: statistical moment method, rare earth, rare earth alloys, melting temperature, vacancy. Classification numbers: 61.66.Dk, 64.30.Ef, 64.70.kd, 64.70.qd. 


\section{INTRODUCTION}

Metals and rare earth alloys are a strategic material for high-tech industries such as electronics, nuclear, optics, superconducting materials, super-magnets, metallurgy... Some materials made from rare earth oxides in nanoscale are used for polishing high precision tools such as optical glasses... [1]. Studying and understanding the mechanical, thermal, electrical, and optical properties of rare earth materials is an urgent need, attracting much attention of scientists. The study of the melting temperature versus pressure is one of the noticeable research directions, especially the thermodynamic properties. Recent studies of $A B$ substitution alloys have shown that a change in the composition of alloy results in a change in the thermodynamic properties and the melting temperature of the alloy. The theoretical and experimental results have shown that the vacancies concentration also influences the melting temperature of the alloy.

There are many methods have been used to study the melting temperature of alloys such as diamond-anvil cell (DAC) [2], ab initio [3], first principle [4], shock-wave experiments [5]. However, these methods have high errors at high pressure and their analytical expressions are complicated. These limitations have made difficulties in numeric calculations. The experimental approaches are costly and difficult to carry out. The statistical moment method used in this study has been expected to solve the current problems.

The purpose of this paper is to study the effects of vacancies on the melting temperature of rare earth substitutional alloys at different pressures. The statistical moment methods [6] were used to find out the analytical expressions to determine the Gibbs free energy needed to create a vacancy. The melting temperature of perfect and defect alloys were also calculated. All calculations were carried out on $\mathrm{AgCe}, \mathrm{Ag}_{2} \mathrm{Ce}$ and $\mathrm{Ag}_{3} \mathrm{Ce}$ alloys.

\section{THEORY}

\section{II.1. The free energy of $\operatorname{defect} A B$ substitutional alloy}

We determine the $A B$ substitutional alloy containing $N\left(N_{A}+N_{B}=N\right)$ atoms and $n$ vacancies $\left(n_{i i} N\right)$. The Gibbs free energy is described by following expression:

$$
G=G_{0}+n^{A} g_{v}^{f A}+n^{B} g_{v}^{f B}-T S_{c}^{n},
$$

where $G_{0}$ is the free energy of the $A B$ substitutional alloy; $g_{v}^{f A}$ is the difference of Gibbs free energy of $A$ metal when it creates one vacancy, $g_{v}^{f B}$ is the difference of Gibbs free energy of $B$ metal when it creates one vacancy and $g_{v}^{f A} \approx \frac{U_{0}^{A}}{2}, g_{v}^{f B} \approx \frac{U_{0}^{B}}{2}$ [7]. $n^{A}$ and $n^{B}$ are vacancies that are created by moving $A$ atom (or $B$ atom) from their lattice point; $U_{0}^{\alpha}$ is the interaction energy, $U_{0}^{\alpha}=\frac{1}{2} \sum_{i} \phi_{i 0}^{\alpha}\left(\left|\vec{a}_{i}\right|\right)(\alpha=A, B)$, and $\phi_{i 0}$ is a paired interaction potential between 0th and $\circlearrowleft_{\leftrightarrow} \hbar$ atoms.

It is assumed that $n^{A}$ is proportional to $N_{A}\left(N_{A}\right.$ is the total numbers of $A$ atoms in $A B$ alloy); $n^{B}$ is proportional to $N_{B}\left(N_{B}\right.$ is the total numbers of $B$ atoms in $A B$ alloy).

$$
\frac{n^{A}}{N_{A}}=\frac{n^{B}}{N_{B}}=\frac{n^{A}+n^{B}}{N_{A}+N_{B}}=\frac{n}{N}
$$

where $n=n^{A}+n^{B}$ is the total of vacancies of $A B$ alloy. The expression (2) should be rewritten as following: 


$$
\begin{aligned}
n^{A} & =n \frac{N_{A}}{N}=n C_{A}, \\
n^{B} & =n \frac{N_{B}}{N}=n C_{B} .
\end{aligned}
$$

Substitute (3) for (1) we get

$$
G=G_{0}+n\left[C_{A} g_{v}^{f A}+C_{B} g_{v}^{f B}\right]-T S_{c}^{n} .
$$

If putting $g_{f}^{A B}=C_{A} g_{v}^{f A}+C_{B} g_{v}^{f B}$, the expression (4) should be rewritten as following:

$$
G=G_{0}+n g_{v}^{f A B}-T S_{c}^{n}=G_{0}+n_{v} N g_{v}^{f A B}-T S_{c}^{n},
$$

From the minimum condition of the Gibbs function, we obtain the expression to calculate the vacancy concentration:

$$
\left(\frac{\partial G}{\partial n_{v}}\right)_{P, T, N}=0 \Rightarrow n_{v}=e^{-\frac{g_{v}^{f A B}}{\theta}}
$$

where $n_{v}=\frac{n}{N+n}$ is the vacancy concentration.

From above expression, we get the expression to calculate the difference of the free Gibbs energy when one vacancy is created in $A B$ substitutional alloy:

$$
g_{f}^{A B}=-\frac{1}{2}\left[C_{A} U_{0}^{A}+C_{B} U_{0}^{B}\right] .
$$

\section{II.2. The melting temperature of $A B$ substitutional alloy at difference pressure}

It is known that the isothermal bulk modulus $B_{T}$ of metals is a function of pressure. Therefore, $B_{T}=B_{T}(P)$ can be written as $[7,8]$ :

$$
B_{T}(P)=B_{0}+B_{0}^{\prime} P+\frac{1}{2} B^{\prime \prime} P^{2}+\ldots
$$

If we only consider for a first-order approximation, $B_{T}$ will be writen as:

$$
B_{T}(P)=B_{0}+B_{0}^{\prime} P
$$

where $B_{0}$ is the isothermal bulk modulus at a pressure $P=0$, and $B_{0}^{\prime}, B_{0}^{\prime \prime}$ are determined by the expressions:

$$
B_{0}^{\prime}=\left(\frac{d B_{T}}{d P}\right)_{P=0} ; \quad B_{0}^{\prime \prime}=\left(\frac{d^{2} B_{T}}{d P^{2}}\right)_{P=0} .
$$

Therefore the ratio of the melting temperature of crystal at pressure that differs from zero and the melting temperature of metal at pressure that equals to zero is written:

$$
\frac{T_{m}(P)}{T_{m}(0)}=\frac{G(P)}{G(0)} \exp \left\{-\int_{0}^{P} \frac{d P^{\prime}}{B\left(P^{\prime}\right)}\right\},
$$

where $G(P)$ is shear modulus at pressure $P \neq 0, G(0)$ is shear modulus at pressure $P=0$.

If we only expand to first order, we will obtain:

$$
\frac{T_{m A B}(P)}{T_{m A B}(0)}=\frac{G(P)}{G(0)} \exp \left\{-\int_{0}^{P} \frac{d P^{\prime}}{B_{0}+B_{0}^{\prime} P^{\prime}}\right\} .
$$


From this, we find out the expression to calculate the melting temperature of $A B$

$$
T_{m A B}(P)=\frac{T_{m A B}(0) B_{0}^{\frac{1}{B_{0}^{\prime}}}}{G(0)} \cdot \frac{G(P)}{\left(B_{0}+B_{0}^{\prime} P\right)^{\frac{1}{B_{0}^{\prime}}}} .
$$

Therefore, if $G(P), G(0), B_{0}, B_{0}^{\prime}$ and $T_{m A B}(0)$ are known, we will determine the melting temperature of perfect $A B$ substitutional alloy at non zero pressure.

The isothermal compressibility coefficient $B_{T}$ of $A B$ substitutional alloy is determined as following [9]:

$$
B_{T, A B}=\frac{1}{\chi_{T, A B}}
$$

where

$$
\begin{gathered}
\chi_{T, A B}=\frac{3\left(\frac{a_{A B}}{a_{0 A B}}\right)^{3}}{2 P+\frac{1}{3 N v_{A B}}\left(\frac{\partial^{2} \psi_{A B}}{\partial a_{A B}^{2}}\right)}, \\
a_{0 A B}=c_{A} a_{0 A} \frac{B_{0 T, A}}{\bar{B}_{0, T}}+c_{B} a_{0 B} \frac{B_{0 T, B}}{\bar{B}_{0, T}}, \\
a_{A B}=c_{A} a_{A} \frac{B_{T, A}}{\bar{B}_{T}}+c_{B} a_{B} \frac{B_{T, B}}{\bar{B}_{T}},
\end{gathered}
$$

where $c_{A}$ and $c_{B}$ are the composition of $A$ metal and $B$ metal in $A B$ substitutional alloy, respectively; $a_{0 \alpha}, a_{\alpha}(\alpha=A, B)$ is in turn the distance between neighbour $\alpha$ atoms at $0(K)$ and $T(K) ; B_{0 T, \alpha}$ and $B_{T, \alpha}$ is the isothermal bulk modulus of $\alpha$ metals at temperature of $T ; \bar{B}_{0 T}$ and $\bar{B}_{T, \alpha}$ are the average of isothermal bulk modulus in $A B$ substitutional alloy at $0(K)$ and $T(K) . \bar{B}_{0 T}$ should be written as following:

$$
\bar{B}_{0 T}=c_{A} B_{T, A}+c_{B} B_{T, B} .
$$

The free energy of $A B$ substitutional alloy is determined by:

$$
\psi_{A B}=c_{A} \psi_{A}+c_{B} \psi_{B}-T S_{n}^{c},
$$

where $\psi_{A}, \psi_{B}$ is free energy of $A$ metal and $B$ metal, respectively.

Shear modulus $G_{A B}$ and $G_{0 A B}$ are determined by the Young modulus $E$ as following [10]:

$$
G_{0 A B}=\frac{E_{0 A B}}{2\left(1+v_{A B}\right)} ; \quad G_{A B}=\frac{E_{A B}}{2\left(1+v_{A B}\right)} ;
$$

where, $v$ is the Poisson coefficient and $E$ is the Young modulus. They are determined by:

$$
\begin{gathered}
v_{A B}=c_{A} v_{A} \frac{B_{T, A}}{\bar{B}_{T}}+c_{B} v_{B} \frac{B_{T, B}}{\bar{B}_{T}}, \\
E_{0 A B}=\frac{1}{\pi a_{0 A B} A_{A B}} ; \quad E_{A B}=\frac{1}{\pi a_{A B} A_{A B}},
\end{gathered}
$$

where $A_{A B}$ in the expression (21) is determined as following:

$$
A_{A B}=c_{A} A_{A} \frac{B_{T, A}}{\bar{B}_{T}}+c_{B} A_{B} \frac{B_{T, B}}{\bar{B}_{T}},
$$

with $A_{\alpha}=\frac{1}{k_{\alpha}}\left[1+\frac{2 \gamma_{\alpha}^{4} \theta^{2}}{k_{\alpha}^{4}}\left(1+\frac{x_{\alpha} \operatorname{coth} x_{\alpha}}{2}\right)\left(1+x_{\alpha} \operatorname{coth} x_{\alpha}\right)\right], \alpha=A, B$. 


$$
\begin{aligned}
& k=\frac{1}{2} \sum_{i}\left(\frac{\partial^{2} \phi_{i 0}}{\partial^{2} u_{i \beta}}\right)_{e q} ; \\
& \gamma=\frac{1}{12} \sum_{i}\left[\left(\frac{\partial^{4} \phi_{i 0}}{\partial u_{i \beta}^{4}}\right)_{e q}+6\left(\frac{\partial^{4} \phi_{i 0}}{\partial^{2} u_{i \beta} \partial^{2} u_{i \gamma}}\right)_{e q}\right],
\end{aligned}
$$

in which $\alpha \neq \beta \neq \gamma ; x=\frac{\hbar \omega}{2 \theta} ; \theta=k_{B} T, \omega=\sqrt{\frac{k}{m}}$.

\section{II.3. Melting temperature of $\operatorname{defect} A B$ substitutional alloy}

It is known that the temperature of crystals is a function of volume $V$, pressure $P$ and balance vacancies concentration. This means $T_{m}=T_{m}\left(P, V, n_{v}\right)$, thus:

$$
T_{m}=T_{m}(P)+\left(\frac{\partial T}{\partial P}\right)_{n_{v}, V} P+\left(\frac{\partial T}{\partial n_{v}}\right)_{V, P} n_{v} .
$$

From the expression calculating the balance vacancies concentration (6), we have:

$$
\begin{aligned}
&\left(\frac{\partial n_{v}}{\partial T}\right)_{P, V}=k_{B}\left(\frac{\partial n_{v}}{\partial \theta}\right)_{P, V} \\
&=k_{B} \frac{\partial}{\partial \theta}\left(\exp \left\{-\frac{g_{v}^{f}}{\theta}\right\}\right)_{P, V} \\
&=k_{B} \exp \left\{-\frac{g_{v}^{f}}{\theta}\right\} \frac{\partial}{\partial \theta}\left(-\frac{g_{v}^{f}}{\theta}\right)_{P, V} \\
&=k_{B} n_{v} \frac{\left(\frac{\partial g_{v}^{f}}{\theta}\right)_{P, V} \theta-g_{v}^{f}}{\theta^{2}} \\
& \Rightarrow\left(\frac{\partial T}{\partial n_{v}}\right)_{P, V}=\frac{\theta^{2}}{k_{B} n_{v}} \cdot\left(\theta \frac{\partial g_{v}^{f}}{\partial \theta}-g_{v}^{f}\right)^{-1} .
\end{aligned}
$$

Substitute (25) to (24), we get the expression to calculate melting temperature of defect $A B$ substitutional alloy [7]:

$$
T_{m A B}^{\text {vacancy }}=T_{m A B}(P)+\left(\frac{\partial T}{\partial P}\right)_{n_{v}, V} P+\frac{T_{m A B}^{2}(P)}{T_{m A B}(0) \frac{\partial g_{v}^{f}}{\partial \theta}-\frac{g_{v}^{f}}{k_{B}}} .
$$

Based on the melting temperature of perfect substitutional alloy, if we know the energy needed to create a vacancy, we will determine the melting temperature of defect substitutional alloy. 


\section{RESULTS AND DISCUSSIONS}

In this paper, we used the Lennard-Jones potential that was written as following [6]:

$$
\phi(r)=\frac{D}{(n-m)}\left[m\left(\frac{r_{0}}{a}\right)^{n}-n\left(\frac{r_{0}}{a}\right)^{m}\right],
$$

where $r_{0}$ is a distance between two atoms corresponding to minimum potential at $D ; n$ and $m$, the numbers determined by experiment corresponding to $A$ metal and $B$ metal. The potential parameters and the Poisson constant of $\mathrm{Ag}, \mathrm{Ce}$ are listed in Table 1.

Table 1. Parameters $D, r_{0}, m, n$ of $\mathrm{Ag}, \mathrm{Ce}$ [11] and the Poisson constant $v$ of $\mathrm{Ag}, \mathrm{Ce}$ [12].

\begin{tabular}{l|lllll}
\hline Metals & $D / k_{B}(K)$ & $r_{0}(\AA)$ & $m$ & $n$ & $v$ \\
\hline \hline $\mathrm{Ag}$ & 5737.19 & 2.8760 & 2.82 & 12.7 & 0.37 \\
\hline $\mathrm{Ce}$ & 8510.85 & 3.6496 & 2.28 & 8.22 & 0.24 \\
\hline
\end{tabular}

In order to carry out numerical calculations, it is necessary to know the factors such as: the isothermal compressibility coefficient $B_{0 T}, B_{T}(13) ; \bar{B}_{0 T}(17)$, the nearest neighbour distances (15), (16), the Poisson coefficient (20), the shear modulus (19) and the Young modulus (21). Based on these the melting temperature of perfect $A B$ substitutional alloy (12), the Gibbs free energy (7) and the melting temperature of defect $A B$ substitutional alloy (26) are calculated.

Table 2. The melting temperature of rare earth alloys at zero pressure.

\begin{tabular}{c|c|c}
\hline Alloys & $T_{m} A B-\operatorname{SMM}(K)$ & $T_{m} A B-\operatorname{Exp}[13](K)$ \\
\hline \hline $\mathrm{AgCe}$ & 1112.2 & 1151.0 \\
\hline $\mathrm{Ag}_{2} \mathrm{Ce}$ & 1191.3 & 1138.0 \\
\hline $\mathrm{Ag}_{3} \mathrm{Ce}$ & 1230.4 & 1263.0 \\
\hline
\end{tabular}

Table 2 is the calculation of the melting temperature results of $\mathrm{AgCe}, \mathrm{Ag}_{2} \mathrm{Ce}$ and $\mathrm{Ag}_{3} \mathrm{Ce}$ at zero pressure by statistical moment method and the experimental results [13]. The results shown that the calculating results by the statistical moment method agreed well with experimental results. The error is less than $5 \%$.

Tables 3, 4 and 5 are the results of melting temperature calculations for perfect and defect alloys. The results show that the melting temperature of alloys increases with the increase in pressure. The melting temperatures of defect alloys of $\mathrm{AgCe}, \mathrm{Ag}_{2} \mathrm{Ce}$ and $\mathrm{Ag}_{3} \mathrm{Ce}$ are always lower than those of perfect alloys. From above tables, when the pressure rises, the melting temperatures of defect alloys approach the melting temperatures value of perfect alloys. The melting temperature of alloys increases with an increase in amount of Ag containing in the alloys because the melting temperature of $\mathrm{Ag}$ higher the melting temperature of $\mathrm{Ce}$. 
Table 3. The pressure dependences of the melting temperature of AgCe alloy.

\begin{tabular}{l|rrrrrrrr}
\hline$P(\mathrm{GPa})$ & 0 & 10 & 20 & 30 & 40 & 50 & 60 & 70 \\
\hline \hline$T_{m} A B$ & 1112.20 & 1250.84 & 1372.06 & 1480.80 & 1580.00 & 1671.64 & 1757.06 & 1837.28 \\
\hline$T_{m} A B^{\text {vacancy }}$ & 1110.25 & 1248.98 & 1370.36 & 1479.32 & 1578.82 & 1670.80 & 1756.62 & 1837.28 \\
\hline
\end{tabular}

Table 4. The pressure dependences of the melting temperature of $\mathrm{Ag}_{2} \mathrm{Ce}$ alloy.

\begin{tabular}{l|rrrrrrrr}
\hline$P(G P a)$ & 0 & 20 & 40 & 60 & 80 & 90 & 100 & 105 \\
\hline \hline$T_{m} A B$ & 1191.28 & 1493.62 & 1743.21 & 1960.85 & 2156.29 & 2247.58 & 2335.24 & 2377.85 \\
\hline$T_{m} A B^{\text {vacancy }}$ & 1188.77 & 1491.09 & 1740.95 & 1959.08 & 2155.21 & 2246.90 & 2335.01 & 2377.85 \\
\hline
\end{tabular}

Table 5. The pressure dependences of the melting temperature of $\mathrm{Ag}_{3} \mathrm{Ce}$ alloy.

\begin{tabular}{l|rrrrrrrrr}
\hline$P(G P a)$ & 0 & 20 & 40 & 60 & 80 & 100 & 120 & 128.5 \\
\hline \hline$T_{m} A B$ & 1230.44 & 1553.45 & 1823.57 & 2061.37 & 2276.67 & 2475.01 & 2660.15 & 2735.53 \\
\hline$T_{m} A B^{\text {vacancy }}$ & 1227.56 & 1550.41 & 1820.67 & 2058.82 & 2274.67 & 2473.73 & 2659.74 & 2735.53 \\
\hline
\end{tabular}

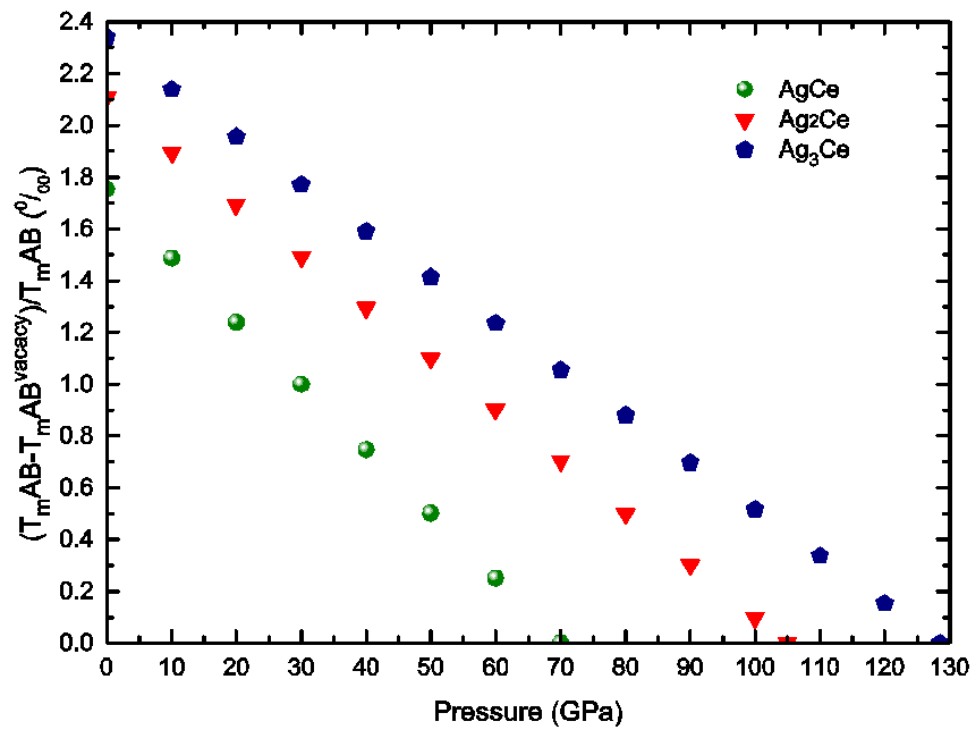

Fig. 1. The effect of vacancies on the melting temperature of $\mathrm{AgCe}, \mathrm{Ag}_{2} \mathrm{Ce}$ and $\mathrm{Ag}_{3} \mathrm{Ce}$ alloys under various pressures.

Fig.1 presents the ratio of $\left(T_{m} A B-T_{m} A B^{\text {vacancy }}\right) / T_{m} A B$ of $\mathrm{AgCe}, \mathrm{Ag}_{2} \mathrm{Ce}$ and $\mathrm{Ag}_{3} \mathrm{Ce}$ alloys under various pressures. This ratio is a function of pressure, the value of this ratio decreases when the pressure increases. It is concluded from the Fig. 1 that the vacancy contribution is negligible 
on the melting temperatures. This contribution accounts for some thousand percent of the melting temperatures at low pressures.

It is also indicated from the Fig. 1 shown that at zero pressure, the impact of vacancy of $\mathrm{AgCe}$ alloy is about $1.75 \% \mathrm{o}, \mathrm{Ag}_{2} \mathrm{Ce}$ is $2.10 \% \mathrm{o}$ and $\mathrm{Ag}_{3} \mathrm{Ce}$ is $2.34 \% \mathrm{o}$. When the pressure increases, the contribution of vacancy gradually decreases, and the impact of vacancy decreases by zero at a definite pressure. For the alloy $\mathrm{AgCe}$ when pressure is $70 \mathrm{GPa}, \mathrm{Ag}_{2} \mathrm{Ce}$ when pressure is $105 \mathrm{GPa}$ and $\mathrm{Ag}_{3} \mathrm{Ce}$ when the pressure is $128.5 \mathrm{GPa}$ the impact of vacancy is zero and that point the melting temperature of defect alloys is equal to the melting temperature of perfect ones.

\section{CONCLUSIONS}

The analytical expressions to calculate the Gibbs free energy needed to create a vacancy and the melting temperature of perfect and defect $A B$ substitutional alloys were found out by the statistical moment method.

The numerical calculations showed that the vacancies influence slightly the melting temperature of alloys. There were no effects of vacancies on the melting temperature of the alloys at high pressure.

\section{REFERENCES}

[1] S. D. Barrett and S. S. Dhesi, The structure of rare-earth metal surfaces, World Scientific, 2001.

[2] S.-N. Luo and D. C. Swift, Physica B: Condensed Matter 388 (2007) 139.

[3] A. B. Belonoshko, S. Simak, A. Kochetov, B. Johansson, L. Burakovsky and D. Preston, Phys. Rev. Lett. 92 (2004) 195701.

[4] Y. Wang, R. Ahuja and B. Johansson, Phys. Rev. B 65 (2001) 014104.

[5] Y. Zhang, T. Sekine, H. He, Y. Yu, F. Liu and M. Zhang, Sci. Rep. 6 (2016) 22473.

[6] N. Tang and V. Van Hung, physica status solidi (b) 149 (1988) 511.

[7] V. Van Hung, D. T. Hai et al., Comput. Mater. Sci. 79 (2013) 789.

[8] L. Burakovsky, D. L. Preston and R. R. Silbar, J. Appl. Phys. 88 (2000) 6294.

[9] D. T. Hai, V. V. Hung and H. P. T. Minh, J. Science HUE 60 (2015) 104.

[10] V. V. Hung and N. T. Hai, Comput. Mater. Sci. 14 (1999) 261.

[11] M. N. Magomedov, High Temperature 44 (2006) 513.

[12] Periodictable, http://periodictable.com/Elements/029/index.html, Accessed: 2018-01-30.

[13] P. Nash and A. Nash, Bull. Alloy Phase Diagr. ' 6 (1985) 350. 